\title{
Variation in chemical constituents of essential oils of the fresh, dried and fermented leaves of Premna serratifolia
}

\author{
Khin Su Yee ${ }^{1,2}$, Penpun Wetwitayaklung1, Worrakanya Narakornwit ${ }^{1}$, \\ Tasamaporn Sukwattanasinit ${ }^{1}$, Bunyapa Wangwattana ${ }^{1}$, Uthai Sotanaphun ${ }^{1 *}$ \\ 1 Department of Pharmacognosy, Faculty of Pharmacy, Silpakorn University, Nakhon Pathom, Thailand \\ Department of Pharmacognosy, University of Pharmacy, Mandalay, Myanmar
}

\begin{abstract}
This study aimed to identify the composition of the essential oils of the fresh, dried and fermented leaves of Premna serratifolia by gas chromatography-mass spectrometry (GC-MS) using DB-5 and Carbowax 20M columns. A total of 77, 82 and 90 compounds were detected, which involved the main compound categories of hydrocarbons, terpenoids, and phenolics. Amyl vinyl carbinol (15.8-32.6\%), linalool (11.1-15.1\%), phytol (7.7$12.5 \%)$, salicylic acid methyl ester (3.9-7.2\%) and $(E)$-caryophyllene (3.1-6.6\%) were predominant components of the fresh leaf oils. After drying and fermentation, the chemical compositions were changed by various reactions. The amounts of amyl vinyl carbinol were decreased to 6.3-13.8\% and 6.9-11.5\% after drying and fermentation, respectively. Likewise, linalool and phytol were decreased to 6.3-7.5\% and 7.3-9.0\% after drying, and decreased to 5.3-7.9\% and 2.0-3.4\% after fermentation, respectively. In both the dried and the fermented leaf oils, alphahumulene was disappeared and beta-myrcene was detected as a new compound. The noticeable changes in chemical composition after drying process were much increasing in the amount of $(E)$-caryophyllene (6.6-12.2\%) to become the most abundant compound, and the hydrolysis of palmitic acid ethyl ester to palmitic acid (5.0\%). The fermentation method could dramatically increase the amounts of phenolic compounds especially $p$ vinylanisole (2.4-41.1\%) which became the major compound, marked decrease the phytol and found acorenone B (4.4\%) as a new compound. The present study demonstrated that drying and fermentation processes affected the volatile composition of the leaves of $P$. serratifolia.
\end{abstract}

Keywords:

Premna serratifolia, Essential oil, GC-MS, Drying, Fermentation

\section{INTRODUCTION}

Premna serratifolia L. (family Lamiaceae) is a shrub or tree in tropical and subtropical regions. Its leaves have a characteristic fetid smell and native people in Celebes use it as a food additive to reduce the fishy smell ${ }^{1}$. In Peninsula, Malaysia, and Indonesia, young leaves of this plant are used as vegetables ${ }^{2}$. In Myanmar, the leaves were used for the treatments of cancer and liver diseases. Some people take the preparation of the fresh leaves and some others use the dried leaves. If the fresh leaves of this plant were kept at room temperature or dried in the shade under inappropriate condition, some leaves will be fermented and turned to dark brown color. The fresh, dried and fermented leaves have different smells which suggested that their volatile chemical constituents should be in variation. Many medicinal plants have been reported for chemical variation between their fresh and dried leaves, such as Tapinanthus bangwensi ${ }^{3}$, Artemisia afra ${ }^{4}$, Ocimum sanctum $^{5}$, and Cymbopogon citratus ${ }^{6}$. One of the most well-known fermented example was the hydrolysis of aroma precursors by endogenous glycosidase during the manufacturing processes of Oolong tea and black tea, and many aroma compounds occurred ${ }^{7}$.

This study was a keen interest to investigate the difference among volatile phytochemical constituents of the fresh, dried and fermented leaves of P. serratifolia

*Corresponding author:

*Uthai Sotanaphun sotanaphun_u@su.ac.th

Pharmaceutical Sciences Asia (C) 2021 by

Faculty of Pharmacy, Mahidol University, Thailand is licensed under CC BY-NC-ND 4.0. To view a copy of this license, visit https:// www.creativecommons.org/licenses/by-nc-nd/4.0/ 
using gas chromatography coupled with mass spectrometry (GC-MS) technique. The result would be declared as basic scientific evidence that their qualities were not equal and should be concerned.

\section{MATERIALS AND METHODS}

\subsection{Plant material}

Leaves of $P$. serratifolia were collected from Mandalay Division, Myanmar, in October 2018. The taxanomic species was identified by comparing its flower and leaf characters with the references ${ }^{8-10}$. The voucher specimen (Ps172018) was deposited with the herbarium of the Department of Pharmacognosy, Silparkorn University, Thailand. The leaves were divided into three parts. The first part was the fresh leaves, the second part was dried in the shade, and the third part was fermented by packing in a plastic bag and place at room temperature for 10 days in the shade until the leaves turned to black color.

\subsection{Preparation of essential oils}

The fresh leaf sample (490 g), the dried leaf sample $(244 \mathrm{~g})$ and the fermented leaf sample (201 g) were separately chopped into small pieces and hydrodistillated in a Clevenger apparatus for 5 hours to obtain essential oils. The collected oils were dried over anhydrous sodium sulfate and stored at $4^{\circ} \mathrm{C}$ in air-tight glass containers. The yields of the essential oils of the fresh, dried and fermented leaves were $0.008,0.139$ and $0.139 \%$, respectively.

\subsection{GC-MS analysis}

The essential oils were analyzed by Agilent 6890 gas chromatography equipped with Agilent technology, 5973N mass selective spectrometric detector (EIMS, electron energy, $70 \mathrm{eV}$, scanning from 40 to $500 \mathrm{~m} / \mathrm{z}$ ) with a quadrupole analyzer and an Agilent Chem Station data system (Agilent Technologies, U.S.A.). Two columns, fused silica capillary column (5\%-phenyl)methylpolysiloxane DB-5 (30 m x $0.32 \mathrm{~mm}$ ID x 0.25 $\mu \mathrm{m}$ film thickness) and Carbowax 20M polyethylene glycol (PEG) $(60 \mathrm{~m} \times 0.25 \mathrm{~mm}$ ID x $0.25 \mu \mathrm{m}$ film thickness) were used. Ultra-high purity helium gas (99.999\%) was used as a carrier gas at a flow rate of $1 \mathrm{~mL} / \mathrm{min}$. The sample $(1.0 \mu \mathrm{L})$ was injected with a splitless mode. The solvent delay for the detector was 3 minutes. The ion source temperature was $230^{\circ} \mathrm{C}$ and quadrupole temperature was programmed at $150^{\circ} \mathrm{C}$. For the DB-5 column, the initial oven temperature was $80^{\circ} \mathrm{C}$ and increased to $130^{\circ} \mathrm{C}$ at the $5^{\circ} \mathrm{C} / \mathrm{min}$, then increased to $280^{\circ} \mathrm{C}$ at the $10^{\circ} \mathrm{C} / \mathrm{min}$ and hold for $5 \mathrm{~min}$. For Carbowax 20M column, the initial oven tempe- rature was $60^{\circ} \mathrm{C}$ and increased to $220^{\circ} \mathrm{C}$ at the $2^{\circ} \mathrm{C} / \mathrm{min}$. Identification of compounds was performed by comparison of their RI relative to $n$-alkanes (C8-C26) with Adams ${ }^{11}$, NIST Chemistry WebBook, SRD $69^{12}$, Babushok et $\mathrm{al}^{13}$ and Leffingwell et $\mathrm{al}^{14}$. Their mass spectra were also compared with libraries databases of Wiley7n.l and NIST 05.

\section{RESULTS AND DISCUSSION}

Essential oils of the fresh leaves of $P$. serratifolia were prepared by hydrodistillation and studied by GC-MS. Two GC columns, the non-polar DB-5, and the polar Carbowax 20M columns were used to ensure the most complete investigation of the constituents. The identification of each compound was based on its mass fragmentation pattern and RI (Retention Index) calculation comparing with data in the references. The results are shown in Table 1 and Table 2. Seventy-seven compounds were detected. Most of the identified compounds were classified into hydrocarbons (26.1\% in DB-5 and 57.1\% in Carbowax 20M), terpenoids $(42.8 \%$ in DB-5 and $26.0 \%$ in Carbowax $20 \mathrm{M})$ and phenolics (15.0\% in DB-5 and $10.9 \%$ in Carbowax 20M) compound categories. Some fatty acids, apocarotenoids, and miscellaneous compounds were also detected (Table 3). The most abundant compounds were amyl vinyl carbinol (15.8\% in DB-5 and $32.6 \%$ in Carbowax 20M), linalool (15.1\% in DB-5 and $11.1 \%$ in Carbowax 20M), phytol (12.5\% in DB-5, and $7.7 \%$ in Carbowax 20M), salicylic acid methyl ester (7.2\% in DB-5 and 3.9\% in Carbowax 20M) and (E)-caryophyllene (6.6\% in DB-5 and $3.1 \%$ in Carbowax 20M). The previous study identified only 4 compounds of eugenol, eugenyl acetate, massoil and cis-2-oxabicyclo, 4.4.0decane in the fresh leaf oil ${ }^{1}$. In this study, a very less amount of eugenol was detected (1.2\% in DB5). This might be due to the different location and climate of plant origin. This study was the first report of the constituent of essential oil obtained from the leaves of this plant growing in Myanmar.

After the leaves of $P$. serratifolia were dried under the shade, increasing in the number of compounds in essential oils was observed, resulting in 82 total compounds; whereas when the leaves were allowed to ferment and turn to dark color, 90 compounds were detected (Tables 1 and Table 2). Some compounds were disappeared and some new compounds occurred as concluded in Figure 1. The compounds those lose after drying and fermentation methods were not much different. The significantly lost compounds were alphahumulene, amyl carbinol, (Z)-3-hexen-1-ol and acetophenone. The new occurring compounds were mostly the small molecular weight terpenoids and aldehyde hydrocarbons compound categories, but they were detected in only trace amounts. 
Table 1. Chemical constituents of the essential oils of the fresh, dried and fermented P. serratifolia leaves analysed by GC-MS using DB-5 column.

\begin{tabular}{|c|c|c|c|c|c|c|c|}
\hline \multirow[t]{2}{*}{ Compound } & \multicolumn{2}{|c|}{ Fresh leaves } & \multicolumn{2}{|c|}{ Dried leaves } & \multicolumn{2}{|c|}{ Fermented leaves } & \multirow{2}{*}{$\begin{array}{l}\text { Literature } \\
\text { RI }\end{array}$} \\
\hline & RI & $\begin{array}{c}\text { \% Relative } \\
\text { amount }\end{array}$ & RI & $\begin{array}{c}\text { \% Relative } \\
\text { amount }\end{array}$ & RI & $\begin{array}{c}\text { \% Relative } \\
\text { amount }\end{array}$ & \\
\hline alpha-Pinene & - & - & 1015 & 0.8 & - & - & $939^{11}$ \\
\hline Amyl vinyl carbinol & 1031 & 15.8 & 1030 & 6.3 & 1031 & 6.9 & $979^{11}$ \\
\hline Amyl ethyl ketone & - & - & - & - & 1034 & 1.2 & $984^{11}$ \\
\hline beta-Myrcene & - & - & 1037 & 8.7 & 1037 & 6.7 & $991^{11}$ \\
\hline Amyl ethyl carbinol & 1049 & 6.4 & - & - & 1040 & 5.7 & $1002^{12}$ \\
\hline Unknown & - & - & - & - & 1048 & 0.7 & - \\
\hline$m$-cymene & 1059 & 0.2 & - & - & - & - & $1026^{12}$ \\
\hline Limonene & 1069 & 0.3 & 1065 & 0.3 & 1066 & 0.4 & $1029^{11}$ \\
\hline Benzeneacetaldehyde & 1079 & 1.5 & - & - & 1076 & 1.0 & $1042^{11}$ \\
\hline Unknown & - & - & - & - & 1115 & 1.2 & - \\
\hline Linalool & 1126 & 15.1 & 1122 & 6.3 & 1123 & 7.9 & $1097^{11}$ \\
\hline (Z)-beta-Terpineol & - & - & - & - & 1125 & 0.6 & $1144^{11}$ \\
\hline Unknown & - & - & - & - & 1144 & 0.4 & - \\
\hline$p$-Vinyl Anisole & 1172 & 4.5 & 1172 & 8.9 & 1184 & 1.4 & $1160^{12}$ \\
\hline neo-Menthol & - & - & 1190 & 0.6 & - & - & $1166^{11}$ \\
\hline Unknown & - & - & - & - & 1190 & 1.1 & - \\
\hline Naphthalene & 1204 & 0.6 & - & - & - & - & $1181^{11}$ \\
\hline Unknown & - & - & - & - & 1205 & 0.5 & - \\
\hline alpha-Terpineol & - & - & - & - & 1208 & 0.7 & $1189^{11}$ \\
\hline Unknown & - & - & 1208 & 5.9 & - & - & - \\
\hline Salicylic acid methyl ester & 1216 & 7.2 & - & - & - & - & $1193^{13}$ \\
\hline beta-Cyclocitral & 1236 & 0.7 & - & - & - & - & $1218^{13}$ \\
\hline Nerol & 1239 & 0.5 & 1237 & 0.3 & - & - & $1230^{11}$ \\
\hline $\begin{array}{l}\text { 5-(1'-1'-Dimethylethyl)bicycle[3,10] hexan- } \\
\text { 2-one }\end{array}$ & - & - & - & - & 1256 & 1.7 & - \\
\hline p-Anisaldehyde & - & - & - & - & 1260 & 1.9 & $1270^{12}$ \\
\hline Unknown & - & - & 1270 & 1.0 & - & - & - \\
\hline alpha-Benzeneacetaldehyde ethylidene- & - & - & 1282 & 0.2 & 1279 & 0.8 & $1279^{12}$ \\
\hline Salicylic acid ethyl ester & 1282 & 1.1 & - & - & - & - & $1311^{12}$ \\
\hline Carbamic acid & - & - & 1307 & 0.7 & - & - & - \\
\hline $\begin{array}{l}\text { 3,4,4a,5,6,8a-Hexahydro-2,5,5,8a- } \\
\text { tetramethyl- (2.alpha.,4a.alpha.,8a.alpha)- } \\
\text { 2H-1-benzopyran }\end{array}$ & 1307 & 0.5 & - & - & - & - & - \\
\hline$p$-Vinylgyaiacol & 1323 & 1.1 & 1322 & 0.1 & 1322 & 0.6 & $1324^{12}$ \\
\hline Benzene,4-ethyl-1,2-dimethoxy- & - & - & - & - & 1329 & 0.6 & - \\
\hline Unknown & - & - & - & - & 1332 & 0.7 & - \\
\hline Acetanisole & - & - & 1361 & 0.1 & 1362 & 0.8 & $1352^{11}$ \\
\hline Eugenol & 1366 & 1.2 & 1365 & 0.7 & 1366 & 1.7 & $1359^{11}$ \\
\hline Unknown & 1369 & 1.3 & 1368 & 0.6 & - & - & - \\
\hline 3,4-Dimethoxystyrene & - & - & - & - & 1371 & 0.6 & $1368^{12}$ \\
\hline Unknown & - & - & - & - & 1384 & 1.4 & - \\
\hline (E)-beta-Damascenone & 1392 & 1.4 & 1392 & 0.7 & - & - & $1385^{11}$ \\
\hline beta-Elemene & 1400 & 0.3 & 1400 & 0.4 & 1400 & 0.6 & $1391^{11}$ \\
\hline Methyleugenol & - & - & 1407 & 0.4 & 1407 & 0.9 & $1404^{11}$ \\
\hline Unknown & - & - & 1413 & 0.4 & - & - & - \\
\hline Isocaryophyllene & - & - & - & - & 1420 & 1.1 & $1423^{12}$ \\
\hline Unknown & - & - & 1421 & 0.7 & - & - & - \\
\hline (E)-Caryophyllene & 1436 & 6.6 & 1438 & 12.2 & 1437 & 12.7 & $1433^{12}$ \\
\hline Dihydro-beta-ionone & - & - & 1447 & 0.4 & - & - & $1444^{12}$ \\
\hline Neryl acetone & - & - & 1456 & 1.0 & 1456 & 0.6 & $1456^{12}$ \\
\hline (E)-beta-Farnesene & 1459 & 0.4 & 1460 & 0.5 & 1460 & 0.6 & $1457^{11}$ \\
\hline alpha-Humulene & 1467 & 1.6 & - & - & - & - & $1463^{12}$ \\
\hline $\begin{array}{l}\text { 1,1,4,8-Tetramethyl-(Z,Z,Z)-4,7,10- } \\
\text { cycloundecatriene- }\end{array}$ & - & - & 1468 & 2.4 & 1468 & 3.0 & - \\
\hline$(E)$-beta-Ionone & 1491 & 0.9 & 1493 & 2.9 & 1493 & 1.7 & $1489^{11}$ \\
\hline Unknown & - & - & - & - & 1499 & 1.1 & - \\
\hline alpha-Farnesene & 1509 & 0.2 & - & - & - & - & $1506^{12}$ \\
\hline beta-Bisabolene & 1514 & 3.0 & 1515 & 3.8 & 1515 & 4.8 & $1512^{12}$ \\
\hline Unknown & - & - & 1529 & 0.8 & 1526 & 1.4 & - \\
\hline beta-Cadinene & - & - & - & - & 1533 & 1.1 & $1539^{11}$ \\
\hline Unknown & - & - & 1534 & 1.4 & - & - & - \\
\hline Nerolidol & 1566 & 0.9 & 1566 & 1.3 & 1567 & 1.3 & $1563^{11}$ \\
\hline
\end{tabular}


Table 1. Chemical constituents of the essential oils of the fresh, dried and fermented P. serratifolia leaves analysed by GC-MS using DB-5 column. (cont.)

\begin{tabular}{|c|c|c|c|c|c|c|c|}
\hline \multirow[t]{2}{*}{ Compound } & \multicolumn{2}{|c|}{ Fresh leaves } & \multicolumn{2}{|c|}{ Dried leaves } & \multicolumn{2}{|c|}{ Fermented leaves } & \multirow{2}{*}{$\begin{array}{c}\text { Literature } \\
\text { RI }\end{array}$} \\
\hline & RI & $\begin{array}{c}\text { \% Relative } \\
\text { amount }\end{array}$ & RI & $\begin{array}{c}\text { \% Relative } \\
\text { amount }\end{array}$ & $\mathbf{R I}$ & $\begin{array}{c}\text { \% Relative } \\
\text { amount }\end{array}$ & \\
\hline Caryophyllene oxide & 1597 & 1.8 & 1598 & 2.6 & 1598 & 2.9 & $1583^{11}$ \\
\hline Humulene oxide & - & - & - & - & 1628 & 0.4 & $1602^{13}$ \\
\hline 3,4-Dimethyl-3-cyclohexen-1-carboxaldehyde & 1629 & 0.3 & - & - & - & - & - \\
\hline beta-Tumerone & - & - & 1674 & 1.0 & - & - & $1669^{11}$ \\
\hline Unknown & - & - & - & - & 1678 & 2.4 & - \\
\hline Unknown & - & - & - & - & 1681 & 2.7 & - \\
\hline Acorenone B & - & - & - & - & 1708 & 4.4 & $1698^{11}$ \\
\hline Myristatic acid ethyl ester & 1794 & 0.3 & - & - & - & - & $1794^{12}$ \\
\hline Isopropyl myristate & 1826 & 0.3 & - & - & - & - & $1824^{12}$ \\
\hline Hexahydrofarnesyl acetone & 1848 & 0.3 & 1844 & 3.6 & 1849 & 1.5 & $1844^{12}$ \\
\hline Isobutyl phthalate & 1875 & 0.2 & - & - & 1875 & 0.3 & $1874^{12}$ \\
\hline Palmitoleic acid methyl ester & - & - & 1912 & 0.9 & 1911 & 0.3 & $1912^{12}$ \\
\hline Farnesyl acetone $\mathrm{C}$ & 1925 & 0.4 & 1926 & 2.0 & 1925 & 0.7 & $1920^{13}$ \\
\hline Isophytol & - & - & - & - & 1951 & 0.1 & $1948^{11}$ \\
\hline (Z)-11-Hexadecenoic acid & - & - & 1958 & 1.8 & - & - & $1953^{12}$ \\
\hline Unknown & 1964 & 1.3 & 1963 & 1.0 & 1963 & 0.8 & - \\
\hline Palmitic acid & - & - & 1978 & 5.0 & - & - & $1968^{13}$ \\
\hline (E)-11-Hexadecenoic acid ethyl ester & 1980 & 0.8 & - & - & 1979 & 0.4 & $1974^{12}$ \\
\hline Palmitic acid ethyl ester & 1990 & 1.2 & - & - & - & - & $1990^{12}$ \\
\hline Geranyl linalool isomer1 & - & - & 2038 & 0.2 & - & - & - \\
\hline Linoleic acid methyl ester & - & - & 2098 & 0.4 & - & - & $2097^{12}$ \\
\hline Linolenic acid methyl ester & - & - & 2106 & 1.2 & 2105 & 0.3 & $2108^{12}$ \\
\hline Phytol & 2128 & 12.5 & 2124 & 7.3 & 2120 & 3.4 & $2122^{12}$ \\
\hline (E)-9-Octadecenoic acid & - & - & 2146 & 0.4 & - & - & $2133^{13}$ \\
\hline Linoleic acid ethyl ester & 2167 & 0.6 & - & - & 2165 & 0.1 & $2164^{12}$ \\
\hline Linolenic acid ethyl ester & 2175 & 2.3 & 2174 & 0.4 & 2173 & 0.6 & $2170^{12}$ \\
\hline Geranyl linalool isomer2 & - & - & 2180 & 0.4 & - & - & - \\
\hline 15-Methyl-heptadecanoic acid ethyl ester & 2194 & 0.4 & - & - & - & - & - \\
\hline$n$-Docosane & - & - & - & - & 2199 & 0.1 & $2200^{14}$ \\
\hline$n$-Tricosane & 2299 & 0.2 & 2299 & 0.1 & 2299 & 0.1 & $2300^{14}$ \\
\hline$n$-Tetracosane & 2399 & 0.1 & 2399 & 0.1 & 2399 & 0.1 & $2400^{14}$ \\
\hline$n$-Pentacosane & 2499 & 0.2 & 2499 & 0.1 & 2499 & 0.2 & $2500^{14}$ \\
\hline Bis-(2-ethylhexyl) phthalate & 2554 & 3.0 & 2552 & 0.1 & - & - & $2556^{14}$ \\
\hline$n$-Hexacosane & 2599 & 0.1 & 2598 & 0.1 & 2598 & 0.2 & $2600^{14}$ \\
\hline$n$-Heptacosane & 2700 & 0.4 & 2699 & 0.4 & 2698 & 0.3 & $2700^{14}$ \\
\hline Squalene & 2826 & 0.2 & - & - & 2825 & 0.1 & $2847^{12}$ \\
\hline$n$-Nonacosane & 2897 & 3.0 & 2898 & 0.4 & 2898 & 0.4 & $2900^{14}$ \\
\hline
\end{tabular}

Table 2. Chemical constituents of the essential oils of the fresh, dried and fermented $P$. serratifolia leaves analysed by GC-MS using Carbowax 20M column.

\begin{tabular}{|c|c|c|c|c|c|c|c|}
\hline \multirow[t]{2}{*}{ Compound } & \multicolumn{2}{|c|}{ Fresh leaves } & \multicolumn{2}{|c|}{ Dried leaves } & \multicolumn{2}{|c|}{ Fermented leaves } & \multirow{2}{*}{$\begin{array}{c}\text { Literature } \\
\text { RI }\end{array}$} \\
\hline & RI & $\begin{array}{c}\text { \% Relative } \\
\text { amount }\end{array}$ & RI & $\begin{array}{c}\text { \% Relative } \\
\text { amount }\end{array}$ & RI & $\begin{array}{c}\text { \% Relative } \\
\text { amount }\end{array}$ & \\
\hline (E)-2-Hexenal & 1271 & 0.6 & 1278 & 0.7 & 1279 & 0.3 & $1216^{12}$ \\
\hline (E)-beta-Ocimene & - & - & 1282 & 0.2 & 1283 & 0.2 & $1250^{12}$ \\
\hline Amyl ethyl ketone & 1299 & 7.8 & 1297 & 0.3 & 1298 & 0.7 & $1264^{12}$ \\
\hline p-Cymene & - & - & 1307 & 0.2 & 1300 & 0.2 & $1270^{13}$ \\
\hline alpha-Terpinolen & - & - & 1308 & 0.1 & - & - & $1282^{13}$ \\
\hline Amyl vinyl ketone & 1325 & 0.3 & 1326 & 1.4 & 1326 & 0.2 & $1301^{13}$ \\
\hline 6-Methyl-5-hepten-2-one & - & - & 1355 & 0.1 & 1355 & 0.1 & $1345^{12}$ \\
\hline Amyl carbinol & 1379 & 5.4 & - & - & - & - & $1371^{12}$ \\
\hline (Z)-3-Hexen-1-ol & 1401 & 2.2 & - & - & - & - & $1373^{13}$ \\
\hline Amyl ethyl carbinol & 1423 & 8.1 & 1414 & 2.1 & 1416 & 6.2 & $1392^{13}$ \\
\hline 3,5,5-Trimethyl-3-cyclohexen-1-one & - & - & 1425 & 0.1 & - & - & $1420^{12}$ \\
\hline Amyl vinyl carbinol & 1474 & 32.6 & 1460 & 13.8 & 1461 & 11.5 & $1444^{13}$ \\
\hline (E)-Linalool oxide & 1476 & 0.2 & - & - & - & - & $1454^{13}$ \\
\hline (Z)-Linalool oxide & 1491 & 0.2 & 1486 & 0.2 & 1486 & 0.3 & $1474^{13}$ \\
\hline$(E, E)-2,4-H e p t a d i e n a l$ & 1502 & 0.1 & 1498 & 0.2 & 1499 & 0.2 & $1491^{13}$ \\
\hline $\begin{array}{l}\text { 3,4,4a,5,6,8a-Hexahydro-2,5,5,8a-tetramethy- } \\
\text { (2.alpha.,4a.alpha.,8a.alpha) 2H-1-benzopyran }\end{array}$ & 1527 & 0.2 & - & - & - & - & - \\
\hline
\end{tabular}


Table 2. Chemical constituents of the essential oils of the fresh, dried and fermented $P$. serratifolia leaves analysed by GC-MS using Carbowax 20M column. (cont.)

\begin{tabular}{|c|c|c|c|c|c|c|c|}
\hline \multirow[t]{2}{*}{ Compound } & \multicolumn{2}{|c|}{ Fresh leaves } & \multicolumn{2}{|c|}{ Dried leaves } & \multicolumn{2}{|c|}{ Fermented leaves } & \multirow{2}{*}{$\begin{array}{c}\text { Literature } \\
\text { RI }\end{array}$} \\
\hline & RI & $\begin{array}{c}\text { \% Relative } \\
\text { amount }\end{array}$ & RI & $\begin{array}{c}\text { \% Relative } \\
\text { amount }\end{array}$ & $\mathbf{R I}$ & $\begin{array}{c}\text { \% Relative } \\
\text { amount }\end{array}$ & \\
\hline Camphor & - & - & 1531 & 0.1 & - & - & $1515^{13}$ \\
\hline (E)-2-Nonenal & - & - & 1538 & 0.2 & 1540 & 0.2 & $1536^{13}$ \\
\hline Linalool & 1554 & 11.1 & 1549 & 7.5 & 1549 & 5.3 & $1543^{13}$ \\
\hline $\begin{array}{l}\text { 1-Methyl-4-(1-methylethyl)-trans-2- } \\
\text { cyclohexen-1-ol }\end{array}$ & - & - & - & - & 1577 & 0.3 & $1571^{12}$ \\
\hline beta-Elemene & - & - & 1578 & 0.2 & - & - & $1574^{12}$ \\
\hline$(E, Z)-2,6-$ Nonadienal & - & - & 1583 & 0.4 & 1585 & 0.6 & $1582^{13}$ \\
\hline (E)-Caryophyllene & 1594 & 3.1 & 1596 & 14.6 & 1597 & 8.4 & $1599^{13}$ \\
\hline Unknown & - & - & - & - & 1603 & 0.6 & - \\
\hline 2-Acetylthiazole & 1632 & 0.1 & - & - & - & - & $1634^{12}$ \\
\hline Acetophenone & 1645 & 2.0 & - & - & - & - & $1648^{12}$ \\
\hline (E)-beta-Farenesene & - & - & - & - & 1651 & 0.3 & $1664^{13}$ \\
\hline$p$-Vinyl anisole & 1659 & 2.4 & 1660 & 13.1 & 1667 & 41.1 & $1670^{12}$ \\
\hline Unknown & 1667 & 0.2 & - & - & 1671 & 0.6 & - \\
\hline 4-Oxoisophorone & - & - & 1694 & 0.2 & 1696 & 0.2 & $1690^{12}$ \\
\hline$(E, E)-2,4$ Nonadienal & - & - & - & - & 1698 & 0.1 & $1696^{13}$ \\
\hline beta-Bisabolene & 1706 & 1.5 & 1707 & 6.0 & 1706 & 2.7 & $1699^{12}$ \\
\hline Naphthalene & 1717 & 0.2 & 1717 & 0.2 & - & - & $1709^{12}$ \\
\hline alpha-Farnesene & - & - & 1722 & 0.4 & 1722 & 0.2 & $1744^{13}$ \\
\hline Unknown & 1722 & 0.2 & - & - & - & - & - \\
\hline delta-Cadinene & - & - & 1737 & 0.2 & - & - & $1756^{13}$ \\
\hline Salicylic acid methyl ester & 1755 & 3.9 & 1751 & 0.5 & 1752 & 0.3 & $1768^{13}$ \\
\hline Salicylic acid ethyl ester & 1788 & 0.4 & - & - & - & - & $1798^{12}$ \\
\hline Nerol & - & - & 1802 & 0.3 & 1801 & 0.1 & $1795^{13}$ \\
\hline Unknown & 1802 & 0.4 & - & - & - & - & - \\
\hline (E)-beta-Damascenone & 1814 & 0.6 & 1813 & 0.7 & 1813 & 0.2 & $1821^{13}$ \\
\hline Dihydro-beta-Ionone & - & - & 1827 & 0.3 & - & - & $1825^{12}$ \\
\hline Unknown & 1830 & 0.2 & - & - & - & - & - \\
\hline Neryl acetone & 1843 & 0.1 & 1844 & 1.2 & 1844 & 0.4 & $1835^{12}$ \\
\hline Geraniol & 1852 & 0.7 & 1851 & 0.7 & 1851 & 0.4 & $1851^{12}$ \\
\hline alpha-Ionone & - & - & 1853 & 0.6 & - & - & $1857^{12}$ \\
\hline 4-Ethyl-1,2-dimethoxy-benzene & - & - & - & - & 1870 & 0.4 & $1875^{12}$ \\
\hline Unknown & 1877 & 0.6 & 1876 & 0.7 & 1877 & 1.1 & - \\
\hline$(E, E, E)-2,4,6$-Nona-trienal & - & - & 1889 & 0.2 & 1889 & 0.4 & - \\
\hline Unknown & 1913 & 0.2 & - & - & - & - & - \\
\hline Unknown & 1923 & 0.2 & - & - & 1923 & 0.5 & - \\
\hline (E)-beta-Ionone & 1943 & 0.2 & 1945 & 2.2 & 1944 & 0.4 & $1936^{13}$ \\
\hline Unknown & 1978 & 0.2 & - & - & - & - & - \\
\hline Caryophyllene oxide & 1991 & 0.7 & 1992 & 2.5 & 1991 & 1.0 & $1986^{13}$ \\
\hline Methyleugenol & - & - & 2002 & 0.7 & 2002 & 0.5 & $2006^{13}$ \\
\hline $\begin{array}{l}\text { 4-(2,2,6-Trimethyl-7- } \\
\text { oxabicyclo[4.1.0]hept-1-yl)-3-buten-2-one }\end{array}$ & 2002 & 0.1 & - & - & - & - & $2002^{12}$ \\
\hline$p$-Anisaldehyde & 2020 & 0.5 & 2020 & 1.7 & 2021 & 1.5 & $2011^{13}$ \\
\hline Nerolidol & 2029 & 0.4 & 2029 & 1.4 & 2029 & 0.4 & $2036^{13}$ \\
\hline Myristatic acid ethyl ester & 2037 & 0.1 & - & - & - & - & $2045^{12}$ \\
\hline Zingiberenol & - & - & - & - & 2114 & 0.1 & $2109^{12}$ \\
\hline Hexahydro farnesyl acetone & 2121 & 0.1 & 2124 & 4.1 & 2122 & 0.8 & $2129^{12}$ \\
\hline Unknown & - & - & - & - & 2100 & 0.2 & - \\
\hline Acetanisole & 2144 & 0.1 & 2144 & 0.4 & 2145 & 0.7 & $2148^{12}$ \\
\hline Eugenol & 2151 & 0.6 & 2151 & 1.5 & 2151 & 1.1 & $2150^{12}$ \\
\hline Longiborneol & - & - & 2160 & 0.4 & 2159 & 0.2 & $2157^{13}$ \\
\hline $\begin{array}{l}\text { 2,6,11,15-Tetramethyl-hexadeca- } \\
\text { 2,6,8,10,14-pentaene }\end{array}$ & 2175 & 0.6 & 2174 & 1.1 & 2174 & 0.4 & - \\
\hline$p$-Vinylguaiacol & 2184 & 0.6 & - & - & 2184 & 0.4 & $2180^{12}$ \\
\hline alpha-Cadinol & 2191 & 0.1 & 2191 & 0.3 & - & - & $2191^{12}$ \\
\hline Unknown & - & - & 2202 & 1.7 & 2202 & 0.5 & - \\
\hline Acorenone B & - & - & - & - & 2217 & 2.4 & - \\
\hline Palmitoleic acid methyl ester & 2226 & 0.1 & 2227 & 0.9 & - & - & $2225^{12}$ \\
\hline Palmitic acid ethyl ester & 2245 & 0.4 & - & - & 2243 & 0.2 & $2235^{12}$ \\
\hline beta-Tumerone & - & - & 2246 & 0.5 & - & - & - \\
\hline 9-hexadecenoic ethyl ester & 2268 & 0.3 & - & - & 2266 & 0.2 & $2269^{12}$ \\
\hline Unknown & - & - & - & - & 2281 & 1.7 & - \\
\hline
\end{tabular}


Table 2. Chemical constituents of the essential oils of the fresh, dried and fermented $P$. serratifolia leaves analysed by GC-MS using Carbowax 20M column. (cont.)

\begin{tabular}{|c|c|c|c|c|c|c|c|}
\hline \multirow[t]{2}{*}{ Compound } & \multicolumn{2}{|c|}{ Fresh leaves } & \multicolumn{2}{|c|}{ Dried leaves } & \multicolumn{2}{|c|}{ Fermented leaves } & \multirow{2}{*}{$\begin{array}{c}\text { Literature } \\
\text { RI }\end{array}$} \\
\hline & RI & $\begin{array}{l}\text { \% Relative } \\
\text { amount }\end{array}$ & RI & $\begin{array}{c}\text { \% Relative } \\
\text { amount }\end{array}$ & RI & $\begin{array}{c}\text { \% Relative } \\
\text { amount }\end{array}$ & \\
\hline Isophytol & - & - & 2284 & 0.3 & - & - & $2293^{13}$ \\
\hline 2,4-Bis(1,1-dimethylethyl)-phenol & 2291 & 0.1 & 2291 & 0.6 & 2291 & 0.2 & $2316^{12}$ \\
\hline $\begin{array}{l}\text { 3,7,11-Trimethyl-(E,E)-2,6,10-dodecatrien-1- } \\
\text { ol }\end{array}$ & - & - & - & - & 2357 & 0.1 & $2366^{13}$ \\
\hline Farnesyl acetone C & 2362 & 0.1 & 2363 & 1.2 & 2362 & 0.4 & $2377^{13}$ \\
\hline Ketole (1H-Indole) & - & - & 2425 & 0.2 & 2425 & 0.2 & $2420^{12}$ \\
\hline Octadecanoic acid ethyl ester & 2453 & 0.1 & - & - & - & - & $2450^{12}$ \\
\hline Olealic acid ethyl ester & 2468 & 0.2 & - & - & - & - & $2461^{12}$ \\
\hline Linoleic acid methyl ester & - & - & 2478 & 0.3 & - & - & $2480^{12}$ \\
\hline Linoleic acid ethyl ester & 2515 & 0.2 & - & - & - & - & $2519^{12}$ \\
\hline Linolenic acid methyl ester & - & - & 2547 & 1.1 & 2546 & 0.2 & $2550^{12}$ \\
\hline Linolenic acid ethyl ester & 2584 & 0.7 & - & - & 2582 & 0.4 & $2594^{12}$ \\
\hline 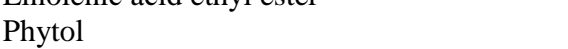 & 2625 & 7.7 & 2620 & 9.0 & 2617 & 2.0 & $2613^{13}$ \\
\hline Dibutyl phthalate & - & - & 2704 & 0.5 & - & - & $2705^{12}$ \\
\hline $\begin{array}{l}\text { 3-(4-Methoxyphenyl),2-propenoic acid } \\
\text { ethyl ester }\end{array}$ & 2641 & 0.5 & - & - & - & - & - \\
\hline
\end{tabular}

Table 3. Category of chemical constituents of the essential oils of the fresh, dried and fermented P. serratifolia leaves.

\begin{tabular}{|c|c|c|c|c|c|c|c|}
\hline \multirow[t]{3}{*}{ Compound category } & \multirow[t]{3}{*}{ Compound sub-category } & \multicolumn{6}{|c|}{ \% Relative amount } \\
\hline & & \multicolumn{2}{|c|}{ Fresh leaves } & \multicolumn{2}{|c|}{ Dried leaves } & \multicolumn{2}{|c|}{ Fermented leaves } \\
\hline & & DB-5 & $\begin{array}{l}\text { Carbowax } \\
\text { 20M }\end{array}$ & DB-5 & $\begin{array}{l}\text { Carbowax } \\
\text { 20M }\end{array}$ & DB-5 & $\begin{array}{l}\text { Carbowax } \\
\text { 20M }\end{array}$ \\
\hline \multirow[t]{11}{*}{ Terpenoids } & Monoterpene hydrocarbons & 0.5 & - & 9.7 & 0.4 & 7.1 & 0.4 \\
\hline & Monoterpene alcohols & 15.6 & 11.8 & 7.1 & 8.5 & 9.2 & 6.1 \\
\hline & Monoterpene ketones & - & - & - & 0.1 & - & - \\
\hline & $\begin{array}{l}\text { Miscellaneous oxygenated } \\
\text { monoterpenes }\end{array}$ & - & 0.4 & - & 0.2 & - & 0.3 \\
\hline & Sesquiterpene hydrocarbons & 12.1 & 4.7 & 19.3 & 21.4 & 23.8 & 11.5 \\
\hline & Sesquiterpenes alcohols & - & 0.1 & - & 0.7 & - & 0.3 \\
\hline & Sesqutiterpene ketones & - & - & 1.0 & 0.5 & 4.4 & 2.4 \\
\hline & $\begin{array}{l}\text { Miscellaneous oxygenated } \\
\text { sesquiterpenes }\end{array}$ & 1.8 & 0.7 & 2.6 & 2.5 & 3.2 & 1.0 \\
\hline & Diterpenoid hydrocarbons & - & 0.6 & - & 1.1 & - & 0.4 \\
\hline & Diterpenoid alcohols & 12.5 & 7.7 & 7.9 & 9.3 & 3.5 & 2.0 \\
\hline & Triterpenoids & 0.2 & - & - & - & 0.1 & - \\
\hline Phenolics & & 15.0 & 10.9 & 10.2 & 18.4 & 7.9 & 6.0 \\
\hline \multirow[t]{2}{*}{ Fatty acids } & Fatty acids & - & - & 7.2 & - & - & - \\
\hline & Fatty acids esters & 5.7 & 2.1 & 2.9 & 2.2 & 1.7 & 1.0 \\
\hline \multirow[t]{4}{*}{ Hydrocarbons } & Hydrocarbon alcohols & 22.2 & 48.2 & 6.3 & 15.9 & 12.5 & 17.8 \\
\hline & Hydrocarbon aldehydes & - & 0.7 & - & 1.7 & - & 1.7 \\
\hline & Hydrocarbon ketones & - & 8.1 & - & 1.6 & 2.9 & 0.9 \\
\hline & Long chain hydrocarbons & 3.9 & - & 1.2 & - & 1.2 & - \\
\hline Apocarotenoids & Apocarotenoids & 4.9 & 1.7 & 11.9 & 12.1 & 5.7 & 2.8 \\
\hline Miscellaneous & & 2.8 & 0.2 & 0.9 & 0.9 & 2.7 & 0.2 \\
\hline Unknowns & & 2.6 & 2.1 & 11.8 & 2.3 & 13.9 & 5.2 \\
\hline
\end{tabular}

The significant new abundant compounds of both the dried and the fermented leaf oils were betamyrcene and (Z,Z,Z)-4,7,10-cycloundecatriene, 1,1,4,8tetramethyl. The major difference between the dried and the fermented leaf oils was the detection of fatty acids (especially palmitic acid) only in the dried leaf oil, whereas acorenone B was found only in the fermented leaf oil.

Changing in a relative amount of each constituent was the major observation after drying or fermentation (Figure 2). Comparing with essential oil of the fresh leaves, essential oils of the dried leaves and the fermented leaves possessed less amounts of monoterpenes (especially linalool), vice versa, a higher proportion of sesquiterpenes (e.g. (E)-caryophyllene, beta-bisabolene, caryophyllene oxide) were observed, especially $(E)$-caryophyllene that became the most abundant compound of the dried leaf oil (6.6-12.2\% in DB-5 and 3.1-14.6\% in Carbowax 20M). The amount of phytol, the only major diterpenoid compound of the fresh leaf oil, was dramatically decreased after fermentation, but changing of this compound after drying was not much significant. In overview, the amount of phenolic compounds did not change much after drying 
while there was a marked increase after fermentation. In detail, the amount of salicylic acid methyl ester decreased in both the dried and the fermented leaf oils, but the amount of $p$-vinyl anisole was dramatically increased after fermentation more than the drying method and became the most abundant compound of the fermented leaf oil (2.4-41.1\% Carbowax 20M). On the other hand, free fatty acids were found only in the dried leaf oil. In overview, the amounts of fatty acid esters (e.g. palmitic acid ethyl ester, linolenic acid ethyl ester) and hydrocarbons (e.g. amyl ethyl carbinol, amyl vinyl carbinol, amyl ethyl ketone) tended to decrease in both the dried and the fermented leaf oils.

The relation between chemical structures of some constituents of the essential oils of the leaves of $P$. serratifolia after drying or fermentation could be explained via different reactions. beta-Myrcene which was the new detected compound of both the dried and fermented leaf oils, possesses the same chemical skeleton of acyclic monoterpenoids as linalool that concomitant dramatically decreasing in relative amount. Dehydration of linalool to form beta-myrcene might be the mechanism of this reaction but it has never been reported in the plant. There was only a report found that linalool could be conversed to beta-myrcene by microbial biotransformation using linalool dehydratase isomerase ${ }^{15}$. After drying and fermentation, alphahumulene was disappeared, whereas relative amounts of $(E)$-caryophyllene and caryophyllene oxide increased. alpha-Humulene and $(E)$-caryophyllene possess a similar sesquiterpenoid skeleton and have humulyl cation as the same biosynthetic precursor ${ }^{16}$. Some unproven factors might affect their biosynthesis expression, and (E)-caryophyllene could be further oxidized to caryophyllene oxide ${ }^{17}$. Most of the fatty acid esters were found mainly in the fresh leaf oil (e.g. palmitic acid ethyl ester), whereas fatty acids were detected only in dried leaf oil (e.g. palmitic acid). In general, fatty acid esters have low boiling points and easily volatilized, then they might be lost during the long period of air dry and fermentation. However, at the same time, some fatty acid esters might be hydrolyzed and caused the formation of fatty acids due to endogenous or microbial enzymes ${ }^{18}$. Hydrolysis of palmitic acid ethyl ester to palmitic acid was the example. As same as fatty acid ester, most of hydrocarbon compounds can easily volatile, then decreasing in the amounts of amyl vinyl carbinol, amyl ethyl carbinol, amyl ethyl ketone was observed. However, some increasing in the amount of amyl vinyl ketone was detected. Functional groups were the only difference among these compounds, and the highest oxidative degree was the functional group of the amyl vinyl ketone. Therefore, the increasing of this compound was possibly due to the oxidation of its derivatives in the fresh leaves.

Apocarotenoids were the known degradative products derived from carotenoids by enzymatic and non-enzymatic oxidation. Increasing in their amounts after drying or fermentation was normally observed in many plant materials such as Morus alba, M. nigra ${ }^{19}$, black tea and Oolong tea ${ }^{20}$. The result of this study was also in the same manner. After drying and fermentation, increasing in their proportion was observed. The relative amounts of apocarotenoids in the fresh leaf oil (4.9\% in DB-5 and $1.7 \%$ in Carbowax 20M) were much increased in the dried leaf oil $(11.9 \%$ in DB-5 and $12.1 \%$ in Carbowax 20M) than fermented leaf oil (5.7\% in DB-5 and 2.8\% in Carbowax 20M). Three significantly increasing compounds were beta-ionone, hexahydrofarnesyl acetone and farnesyl acetone C. Degradation mechanisms of carotenoids to form these three compounds have already been reported ${ }^{19,21}$.

Biological activities of some major compounds of essential oils of $P$. serratifolia have been reported and supported the traditional usage of anticancer and the treatment of liver diseases. Linalool have been reported for antimicrobial, anti-inflammatory, anticancer, and antioxidant properties ${ }^{22}$. (E)-caryophyllene, palmitic acid, phytol, apocarotenoids and amyl vinyl carbinol possessed anticancer, and antioxidant activities ${ }^{23-27}$. beta-Myrcene was antiproliferative compound ${ }^{28}$, whereas $p$-vinylanisole and methyl salicylate were shown to have antioxidant activity ${ }^{29-30}$. However, after drying or fermentation, amounts of some compounds increased and some compounds decreased. Therefore, biological activities of $P$. serratifolia leaves after drying or fermentation should be varied in potency from the fresh leaves and should be studied in more detail.

\section{CONCLUSIONS}

After drying and fermentation, a significant change in the chemical composition of essential oil of the leaves of $P$. serratifolia was detected. Some compounds disappeared, some new compounds occurred, and most compounds changed in their relative amounts. Dehydration, hydrolysis, and oxidation were suggested as the transformation reactions of some compounds. This result indicated the importance of the post-harvesting process on the quality of this herb. Bioactivity of its fresh, dried and fermented leaves would be further studied.

\section{ACKNOWLEDGEMENT}

We would like to thank Faculty of Pharmacy, Silpakorn University, Thailand, for providing chemical, glassware, equipment, and supporting laboratory facilities used this study.

\section{Conflict of interest}

None to declare. 


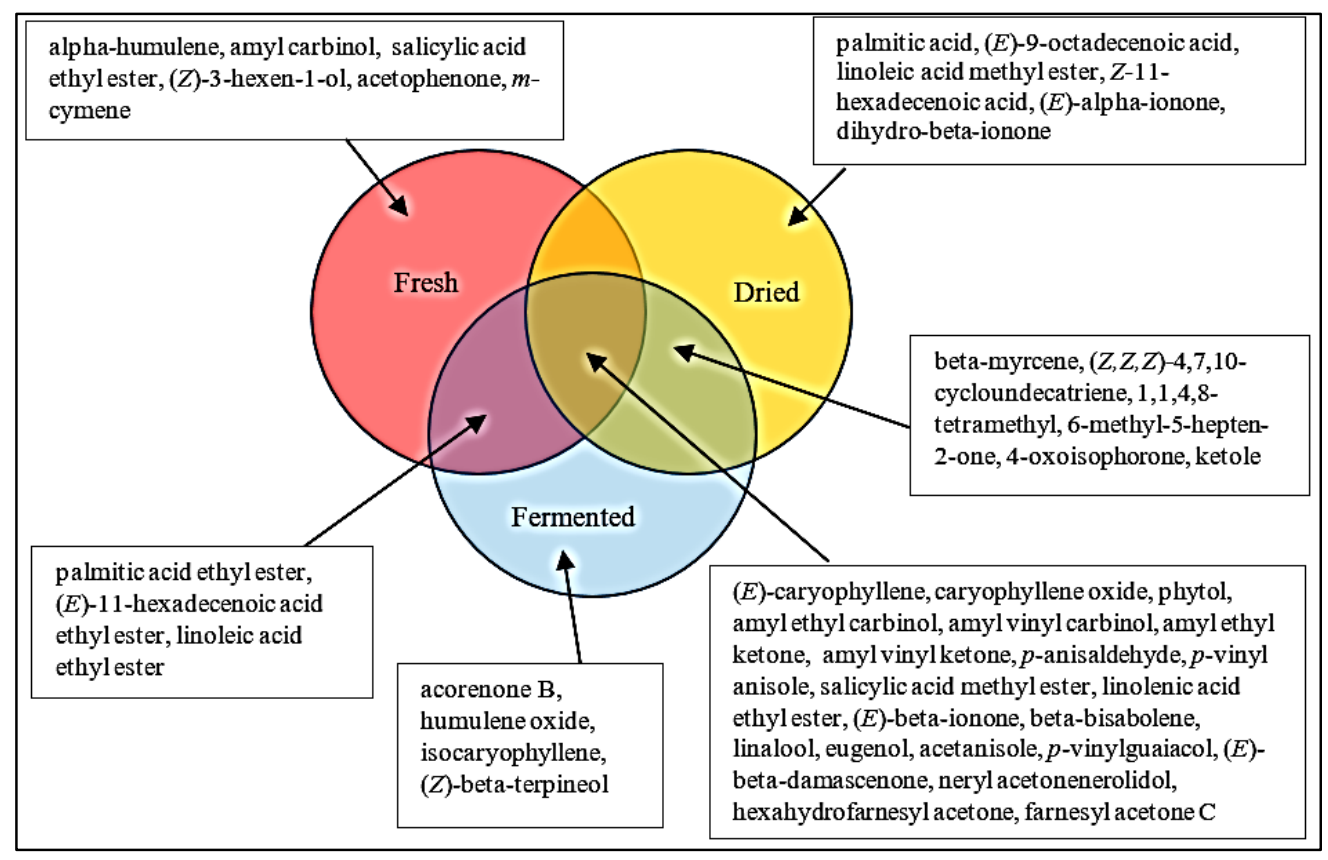

Figure 1. Comparison of some constituents of the essential oils of the fresh, dried and fermented P. serratifolia leaves.

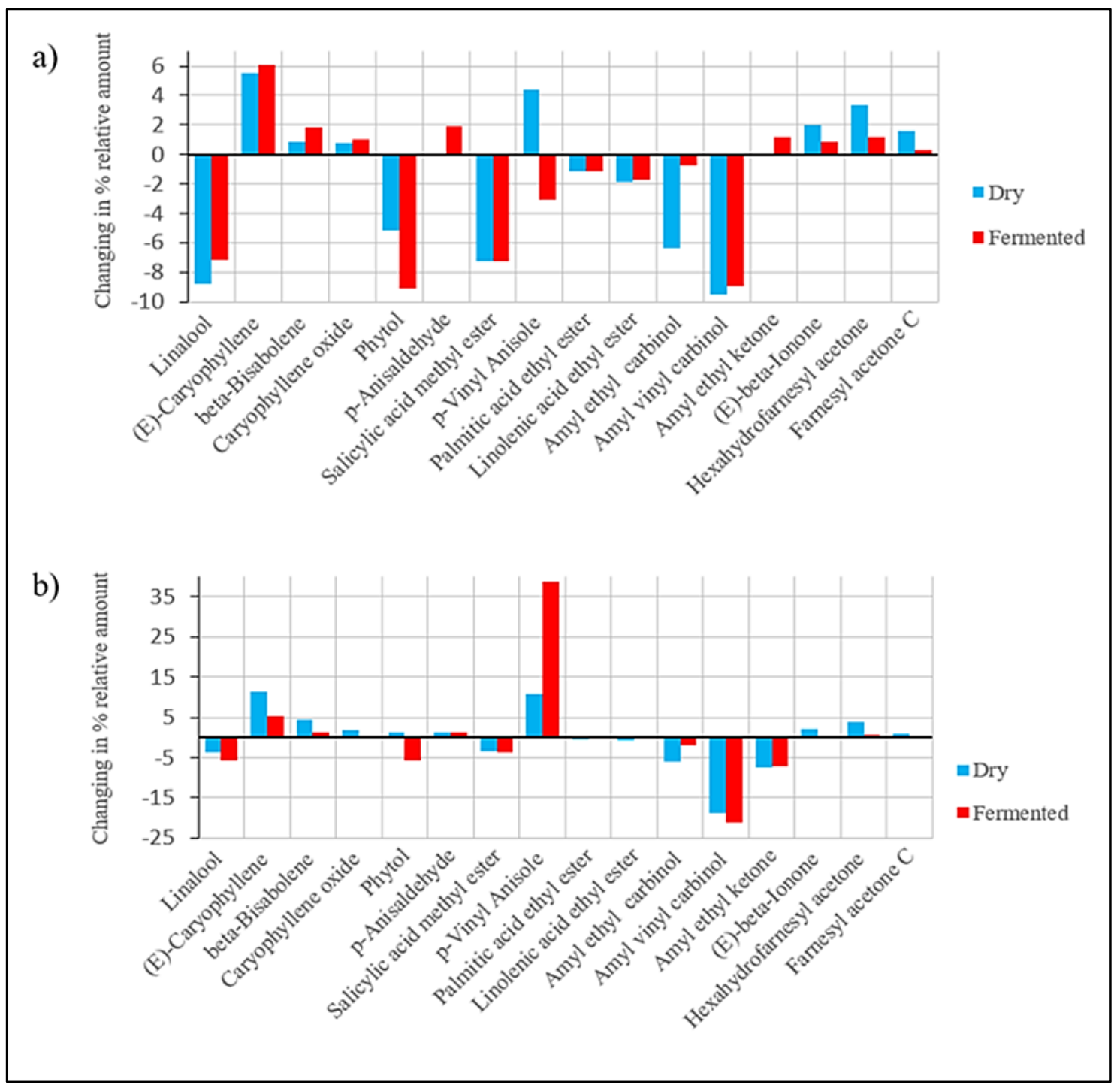

Figure 2. Changing in relative amounts of some constituents of the essential oils of the leaves of $P$. serratifolia after drying and fermentation, analysed by DB-5 (a) and Carbowax 20M (b) columns. 


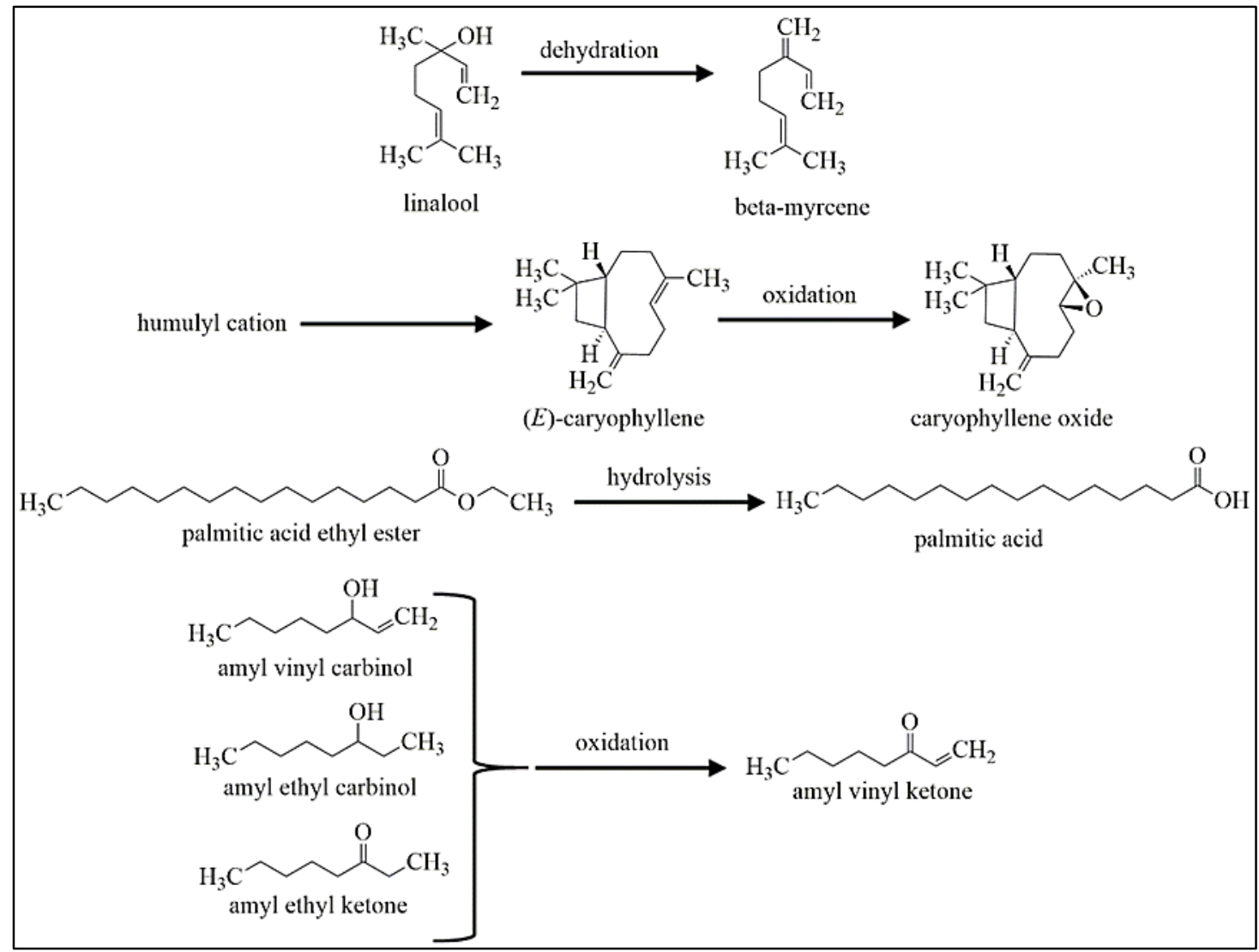

Figure 3. Proposed transformation mechanisms of some volatile compounds of the leaves of $P$. serratifolia after drying and fermentation.

\section{Funding}

None to declare.

\section{Ethics approval}

None to declare.

\section{Article info:}

Received April 17, 2020

Received in revised form July 28, 2020

Accepted December 9, 2020

\section{REFERENCES}

1. Nurliana L, Musta R, Rudi L. Microencapsulation of essential oil from rogo plant (Premna serratifolia L.) as antibactery Escherichia coli. Eng Sci Technol. 2018;7:314-23.

2. de Kok R. The genus Premna L.(lamiaceae) in the flora Malesiana area. Kew Bull. 2013;68(1):55-84.

3. Atewolara-Odule OC, Oladosu IA. Comparison of chemical compositions of essential oils from the fresh and dried leaves of Tapinanthus bangwensis (Engl. and K. Krause) Danser [Loranthaceae]. Am J Essent Oil Nat Prod. 2016;4(3):31-3.

4. Adeogun OO, Maroyi A, Afolayan AJ. Variation in the chemical composition of essential oils from Artemisia afra (Jacq) ex-wild leaf obtained by different methods and the effect of oil extracts on Artemia salina L. Trop J Pharm Res. 2018;17(3):519-28.

5. Mirdha B, Naik S, Mahapatra S. Antimicrobial activities of essential oils obtained from fresh and dried leaves of Ocimum sanctum (L.) against enteric bacteria and yeast. Acta Hortic. 2007;756:267-70.

6. Hanaa AM, Sallam Y, El-Leithy A, Aly SE. Lemongrass (Cymbopogon citratus) essential oil as affected by drying methods. Ann Agric Sci. 2012;57(2):113-6.

7. Baldermann S, Yang Z, Katsuno T, Tu VA, Mase N, Nakamura
Y, et al. Discrimination of green, Oolong, and black teas by GC-MS analysis of characteristic volatile flavor compounds. Am J Anal Chem. 2014;5:620-32.

8. Shou-liang C, Gilbert MG. Verbenaceae. In: Wu Z, Raven PH, editors. Flora of China, Volume17: Verbenaceae through Solanaceae. Missouri Botanical Garden Press; 1994. p. 1-49.

9. Munir AA. A taxonomic revision of the genus Premna L. (Verbenaceae) in Australia. J Adel Bot Gard. 1984;7(1):1-43.

10. Leeratiwong C, Chantaranothai P, Paton AJ. A synopsis of the genus Premna L.(Lamiaceae) in Thailand. Trop Nat Hist. 2009; 9(2):113-42.

11. Adams RP. Identification of Essential Oil Components by Gas Chromatography/Mass Spectrometry. $4^{\text {th }}$ ed. Allured Publishing Corporation: Carol Stream; 2007.

12. National Institute of Standard and Techonology Chemistry WebBook, SRD 69; 2018. [cited 2018 October]. Available from: http://www.webbook.nist.gov.

13. Babushok V, Linstrom P, Zenkevich I. Retention indices for frequently reported compounds of plant essential oils. J Phys Chem Ref Data. 2011;40(4):1-15.

14. Leffingwell JC, Alford E, Leffingwell D, Penn R, Mane S. Identification of the volatile constituents of Cyprian Latakia tobacco by dynamic and static headspace analyses. Leffingwell Rep. 2013;5(2):1-29.

15. Nestl BM, Geinitz C, Popa S, Rizek S, Haselbeck RJ, Stephen $\mathrm{R}$, et al. Structural and functional insights into asymmetric enzymatic dehydration of alkenols. Nat Chem Biol. 2017;13(3): 275-81.

16. Yu F, Okamto S, Nakasone K, Adachi K, Matsuda S, Harada H, et al. Molecular cloning and functional characterization of $\alpha$ humulene synthase, a possible key enzyme of zerumbone biosynthesis in shampoo ginger (Zingiber zerumbet Smith). Planta. 2008;227(6):1291-9.

17. Turek C, Stintzing FC. Stability of essential oils: a review. Compr Rev Food Sci F. 2013;12(1):40-53. 
18. Mozafari AA, Vafaee Y, Shahyad M. Phytochemical composition and in vitro antioxidant potential of Cynodon dactylon leaf and rhizome extracts as affected by drying methods and temperatures. J Food Sci Tech. 2018;55(6):2220-9.

19. Radulović NS, Miljković VM, Mladenović MZ, Nikolić GS. Essential oils of Morus alba and M. nigra leaves: Effect of drying on the chemical composition. Nat Prod Commun. 2017; 12(1):115-18.

20. Ho C-T, Zheng X, Li S. Tea aroma formation. Food Sci Hum Wellness. 2015;4(1):9-27.

21. Bonne T. Oxidation and thermal degradation of carotenoids. J Oil Palm Res. 1999;2:62-78.

22. Kamatou GP, Viljoen AM. Linalool-A review of a biologically active compound of commercial importance. Nat Prod Commun. 2008;3(7):1183-92.

23. Dahham SS, Tabana YM, Iqbal MA, Ahamed MB, Ezzat MO, Majid AS, et al. The anticancer, antioxidant and antimicrobial properties of the sesquiterpene $\beta$-caryophyllene from the essential oil of Aquilaria crassna. Molecules. 2015;20(7): 11808-29.
24. Ravi L, Krishnan K. Cytotoxic potential of $N$-hexadecanoic acid extracted from Kigelia pinnata leaves. Asian J Cell Biol. 2017;12:20-7.

25. Islam MT, Ali ES, Uddin SJ, Shaw S, Islam MA, Ahmed MI, et al. Phytol: A review of biomedical activities. Food Chem Toxicol. 2018;121:82-94.

26. Sharoni Y, Linnewiel-Hermoni K, Khanin M, Salman H, Veprik A, Danilenko M, et al. Carotenoids and apocarotenoids in cellular signaling related to cancer: A review. Mol Nutr Food Res. 2012;56(2):259-69.

27. Al-Fatimi M, Wurster M, Lindequist U. Chemical composition, antimicrobial and antioxidant activities of the volatile oil of Ganoderma pfeifferi Bres. Medicines (Basel). 2016;3(2):10.

28. Blowman K, Magalhães M, Lemos MFL, Cabral C, Pires IM. Anticancer properties of essential oils and other natural products. Evid Based Complementary Altern Med. 2018(7);1-12.

29. Pereira DM, Valentão P, Pereira JA, Andrade PB. Phenolics: From chemistry to biology. Molecules. 2009;14:2202-11.

30. Oloyede GK. Toxicity, antimicrobial and antioxidant activities of methyl salicylate dominated essential oils of Laportea aestuans (Gaud). Arab J Chem. 2016;9:840-45. 